tetraethylenepentamine: a phase I/II clinical trial. Bone Marrow Transplant 2008; 41: 771-778.

7 de Lima MR, McNiece I, Robinson SN, Munsell M, Eapen M, Horowitz M et al. Cord blood engraftment with ex vivo mesenchymal-cell coculture. N Engl J Med 2012; 367: 2305-2315.

8 Horowitz M, Stiff PJ, Chao NJ, Rizzieri D, Long G, Sullivan K et al. Nicord ${ }^{\mathbb{R}}$ expanded hematopoietic progenitor cells (HPC) are capable of outcompeting the unmanipulated (UM) cord blood unit and of prolonged myeloid and lymphoid engraftment following myeloablative dual umbilical cord blood (UCB) transplantation. Biol Blood Marrow Transplant 2013; 19: Abstract 18.

9 Wagner JE, Brunstein CG, McKenna D, Sumstad D, Maahs S, Boitano AE et al. Safety and exploratory efficacy of ex-vivo expanded umbilical cord blood (UCB) hematopoietic stem and progenitor cells (HSPC) using cytokines and Stem-Regenin 1 (SR1): Interim results of a phase 1/2 dose escalation clinical study. Blood 2013; 122: Abstract 698.
10 Sang L, Coller HA, Roberts JM. Control of the reversibility of cellular quiescence by the transcriptional repressor HES1. Science 2008; 321: 1095-1100.

11 Majeti R, Park CY, Weissman IL. Identification of a hierarchy of multipotent progenitors in human cord blood. Cell Stem Cell 2007; 1: 635-645.

12 Manz MG, Miyamoto T, Akashi K, Weissman IL. Prospective isolation of human clonogenic common myeloid progenitors. Proc Natl Acad Sci USA 2002; 99: 11872-11877.

13 Thomsen JS, Kietz S, Ström A, Gustafsson J. HES-1, a novel target gene for the aryl hydrocarbon receptor. Mol Pharmacol 2004; 65: 165-171.

14 Kunisato A, Chiba S, Nakagami-Yamaguchi E, Kumano K, Saito T, Masuda S et al. HES-1 preserves purified hematopoietic stem cells ex vivo and accumulates side population cells in vivo. Blood 2003; 101: 1777-1783.

15 Singh KP, Garrett RW, Casado FL, Gasiewicz TA. Aryl hydrocarbon-receptor null allele mice have hematopoietic stem/progenitor cells with abnormal characteristics and functions. Stem Cells Dev 2011; 20: 769-784.

\title{
OPEN
}

\section{Effects of the small-molecule inhibitor of integrin $\alpha 4$, TBC3486, on pre-B-ALL cells}

Leukemia (2014) 28, 2101-2104; doi:10.1038/leu.2014.182

The treatment of patients with chemotherapy-resistant leukemia remains a challenge. A role of the microenvironment for drug resistance of leukemia cells has been proposed. ${ }^{1}$ We have identified the adhesion molecule integrin $\alpha 4$ as a central mediator of drug resistance of pre-B-cell acute lymphoblastic leukemia (ALL). ${ }^{2}$ We thus demonstrated that chemotherapyresistant pre-B-ALL cells can be eradicated in a xenograft model by concurrent blockade of $\alpha 4$ using natalizumab, a humanized anti- $\alpha 4$ antibody in clinical use against multiple sclerosis, ${ }^{3}$ and Crohn's Disease. ${ }^{2}$ Here, we extended our studies to an alternative $\alpha 4$ inhibitor, the non-peptidic small molecule TBC3486. Previous in vitro assays and molecular modeling studies indicated that TBC3486 behaves as a ligand mimetic, competing with VCAM-1 for the MIDAS site of integrin $\alpha 4{ }^{4}$ As such, the compound has shown efficacy in integrin $\alpha 4$-dependent models of inflammatory and autoimmune disease ${ }^{4}$ and has shown efficacy in mice with autoimmune encephalomyelitis, a model for multiple sclerosis. ${ }^{5}$ As opposed to natalizumab, which will inhibit both members of the $\alpha 4$ integrin family, $\alpha 4 \beta 1$ and $\alpha 4 \beta 7$, TBC3486 is 200 -fold more potent in inhibiting $\alpha 4 \beta 1$ than $\alpha 4 \beta 7$. In addition, it is completely inactive against all other integrins tested, including members of the $\beta 2, \beta 3$ as well as other members of the $\beta 1$ family of integrins. ${ }^{4}$ The potential usefulness of this novel inhibitor for pre-B-ALL treatment was tested in our established in vitro and in vivo assays. $^{2,4}$ We evaluated the effect of TBC3486 on de-adhesion of patient-derived ALL cells (LAX7R) using established adhesion assays. As a control for our studies, a close structural analog was used that lacks activity toward $\alpha 4 \beta 1$ integrin (THI0012). After

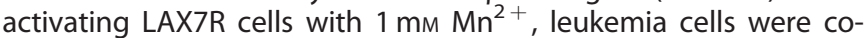
cultured with the murine stromal cell line OP9., ${ }^{6,7}$ Subsequently, LAX7R cells were treated with different doses of $\operatorname{TBC} 3486(5,10$ and $25 \mu \mathrm{M})$ and its control, THI0012 (5, 10 and $25 \mu \mathrm{M})$, for 4 days. TBC3486 dose-dependently inhibited adhesion of ALL cells (Figure 1a), albeit the adhesion was not completely blocked. The dose of $25 \mu \mathrm{m}$ was selected for subsequent studies. The concentrations of compound required for inhibition in these assays are higher than previously reported. ${ }^{4}$ This is due to the fact that TB3486 is highly protein bound in the presence of $20 \%$ serum (used in these assays), which significantly reduces the amount of free compound available to bind to the integrin receptor. Next, we determined whether TBC3486 decreases binding of three xenograft cells derived from primary pre-B-ALL cases (LAX7R, ICN3 and SFO3) to the counter-receptor of $\alpha 4$ integrin, human VCAM-1. Adhesion assays were performed as previously described $^{4,8,9}$ by culturing ALL samples treated with TBC3486 $(25 \mu \mathrm{M})$ or THI0012 $(25 \mu \mathrm{M})$ on hVCAM-1-coated plates for 2 days. Compared with the control group, TBC3486-treated ALL cells showed significantly less adhesion to hVCAM-1 (Figures 1c, e and g); however, the adhesion was not completely blocked. CD49d (MFI) is expressed with higher intensity in LAX7R compared with the other two samples (ICN3 and SFO3) (data not shown), which may explain why TBC3486 blocked a larger percentage of LAX7R adhesion to VCAM-1. In addition to blocking cell adhesion, TBC3486 treatment also specifically targeted the expression of integrin $\alpha 4$, but not integrin $\alpha 5$ and $\alpha 6$ (Figure $1 \mathrm{~b}$ ). The treatment with TBC3486 did not affect cell viability in all three cases (Figures $1 \mathrm{~d}, \mathrm{f}$ and $\mathrm{h}$ ) compared with the THI0012 control. Taken together, TBC3486 leads to the partial de-adhesion of pre-B-ALL cells from its counter-receptor VCAM-1 under the conditions described.

We previously showed that antibody-mediated integrin $\alpha 4$ blockade can sensitize leukemia cells to chemotherapy. ${ }^{2}$ Therefore, we evaluated whether TBC3486 can not only de-adhere cells from its counter-receptors but also sensitize them to chemotherapy. To study the drug sensitivity of human leukemia, ALL (LAX7R) cells were co-cultured with murine calvaria-derived OP9 stromal cells, which allow in vitro studies beyond 2 days. Then this co-culture was exposed to chemotherapy as previously described. ${ }^{9,10}$ ALL cells were treated with chemotherapy (vincristine, dexamethasone, L-asparaginase; VDL) or saline as control for 4 days, with TBC3486 $(25 \mu \mathrm{M})$ or control THI0012. TBC3486 with VDL treatment reduced the cell viability of human leukemia LAX7R $(51 \pm 1 \%$ vs $31 \pm 1 \%$; $P<0.05)$ (Figure $2 a)$, ICN3 $(60 \pm 3 \%$ vs $49 \pm 1 \% ; P<0.05)$ (Figure $2 b)$ and SFO3 (78 $\pm 1 \%$ vs $65 \pm 1 \% ; P<0.05$ ) (Figure $2 \mathrm{c}$ ), indicating that this combination therapy sensitized leukemia cells to chemotherapy in vitro. Nevertheless, all chemoprotection afforded by OP9 cells was removed by TBC3486 in spite of only partial de-adhesion. This observation at least suggests that effects over and above adhesion and physical chemoprotection provided by stromal cells are mediated through $\alpha 4$ integrin. 


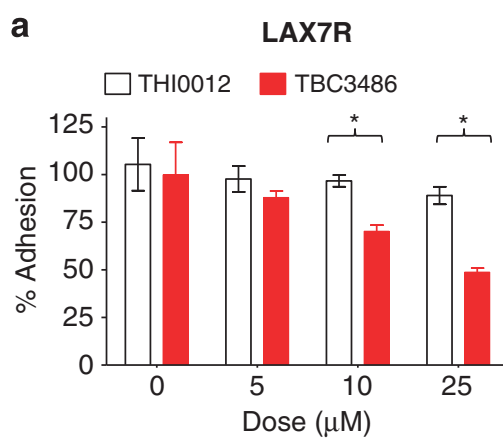

C

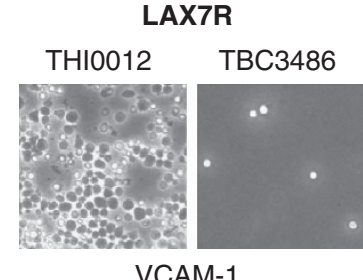

VCAM-1

e

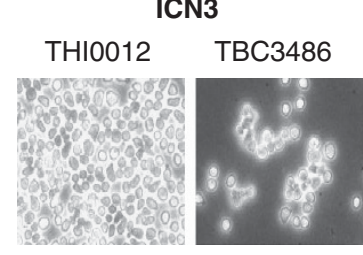

VCAM-1

g

SF03

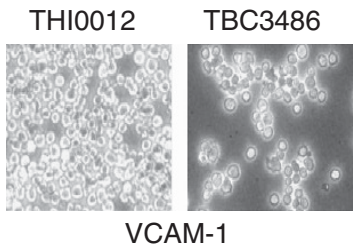

b

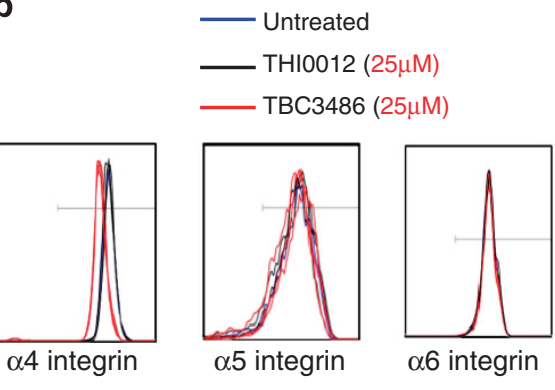

d
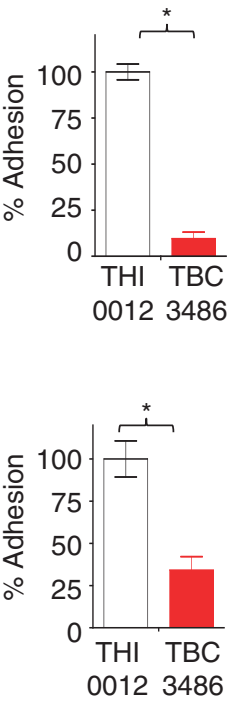

h

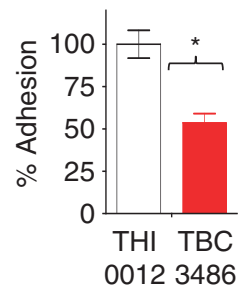

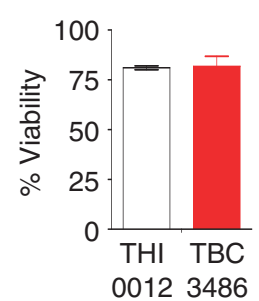

f
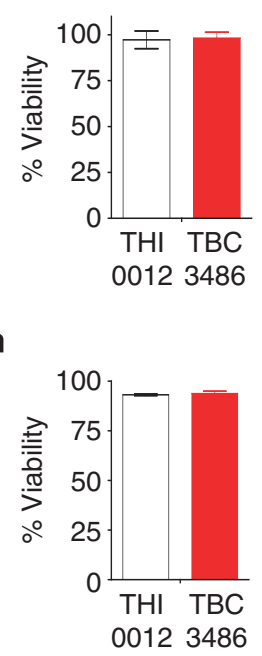

Figure 1. Effects of TBC3486 treatment on adhesion and integrin expression of ALL cells. (a) Percentage of adhesion of LAX7R cells treated with different doses of TBC3486 and THI0012 on OP9 for 4 days. ${ }^{*} P<0.05$, mean \pm s.d., performed in triplicates. (b) Expression of $\alpha 4, \alpha 5$ and $\alpha 6$ integrins presented as histograms after 4 days of TBC3486 (25 $\mu \mathrm{m})$ and THI0012 (25 $\mu \mathrm{m})$ treatment. Three ALL cells (c,d: LAX7R; e,f: ICN3; g,h: SF03) were treated with THI0012 control (white bar) or TBC3486 (red bar) on plates coated with human VCAM-1 or $2 \%$ bovine serum albumin as control for 2 days. (c, e, g) Adhesion of ALL cells ( $\times 400$ magnification) and $\%$ of adhering cells is shown. (d, f, h) Viability of leukemia cells treated with TBC3486 or THI0012 control was determined by trypan blue exclusion.

To determine whether TBC3486 can prolong the survival of xenotolerant mice bearing human leukemia cells in vivo, LAX7R cells were injected into NOD/SCID hosts. Three days after leukemia cell transfer, mice received either TBC3486 or THI0012 (control) $(10 \mathrm{mg} / \mathrm{kg} / \mathrm{d})$ daily for 2 weeks with two daily intraperitoneal injections to ensure stable plasma levels, with or without VDL chemotherapy. TBC3486-treatment $(n=7)$ resulted in prolonged survival time compared with THI0012 control mice $(n=3)$ (median survival time (MST) $=41$ vs 33 days, $P=0.001)$. In combination with chemotherapy, the Kaplan-Meier survival curve indicated that TBC3486+VDL-treatment $(n=6)$ resulted in a significantly prolonged survival time (compared with the VDL + THI0012 control $(n=7)$ (MST $=82$ vs 58 days, $P=0.0003$; Figure $2 d$ ).

TBC3486 preferentially inhibits the high-affinity form of the integrin $\alpha 4{ }^{4}$ It may thus interfere with the function of lymphocytes entertaining the inflammatory response in multiple sclerosis, but may not interfere with that of lymphocytes causing inflammatory bowel disease. Which $\beta$-integrin is the more relevant partner for $\alpha 4$ in the context of drug resistance in ALL is unclear. In addition, TBC3486 mimics VCAM-1 binding, inducing the highaffinity conformation of integrin $\alpha 4$; by contrast, natalizumab recognizes and blocks the ligand-binding moiety of $\alpha 4$ in any conformation and without affecting activation status. Whether, therefore, TBC3486-binding of integrin $\alpha 4$ might elicit outside-in signaling would have to be addressed in further studies. Although unknown off-target effects of the compound cannot be excluded, it should be emphasized that the control compound, THI0012, is structurally identical to TBC3486 except it is the opposite enantiomer. This simple change in stereochemistry eliminates its activity toward $\alpha 4 \beta 1$. The fact that this control compound has no 
a

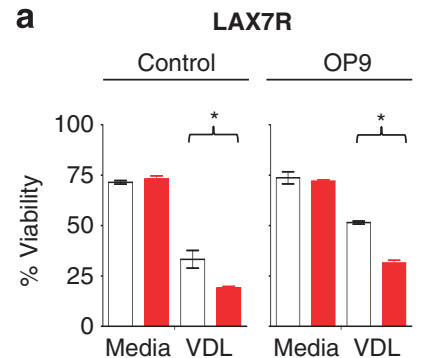

b

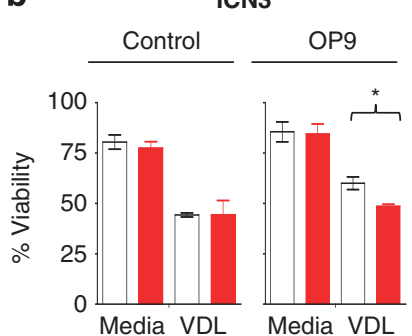

C

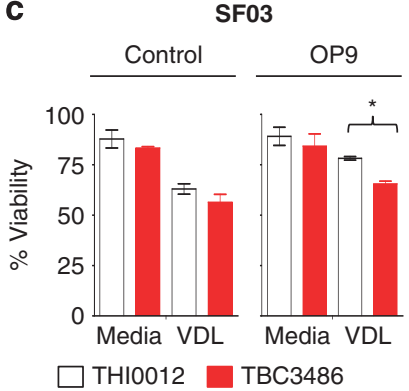

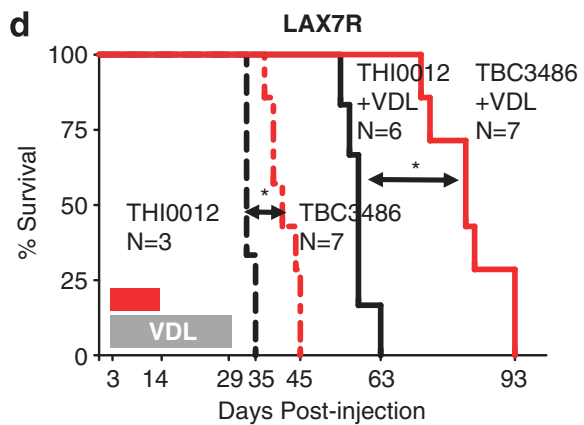

Figure 2. TBC3486 treatment sensitizes ALL cells to chemotherapy. (a-c) ALL cells (a) LAX7R; (b) ICN3; (c) SF03) were co-cultured with (right

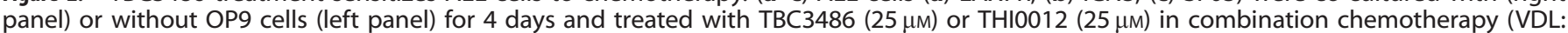
$\mathrm{V}=0.0005 \mu \mathrm{M}, \mathrm{D}=0.005 \mathrm{~nm}, \mathrm{~L}=0.0005 \mathrm{IU} / \mathrm{ml})$ in vitro and viability of leukemia cells was determined by trypan blue exclusion $* P<0.05$; experiments were performed in triplicates. (d) ALL cells (LAX7R) were injected into NSG mice $\left(5 \times 10^{4}\right.$ cells/mouse) and recipient mice were then treated with $10 \mathrm{mg} / \mathrm{kg} /$ day TBC3486 or THI0012 control, starting on day 3 post leukemia injection. The daily administrations were split into two intraperitoneal injections per day for 2 weeks. In addition, mice were treated with vincristine (0.5 mg/kg once a week), dexamethasone $(8 \mathrm{mg} / \mathrm{kg}$ five times a week) and L-asparaginase $(800 \mathrm{lU} / \mathrm{kg}$ five times a week) for 4 weeks. Kaplan-Meier survival curve was analyzed and MST was calculated for each group: THI0012 $(n=3$, MST $=33$ days) (black dashed); TBC3486 $(n=7$, MST $=41$ days) $($ red dashed); THI0012 + VDL $\left(n=6, \mathrm{MST}=58\right.$ days) (black solid); TBC3486 + VDL $\left(n=7, \mathrm{MST}=82\right.$ days) (red solid). ${ }^{*} P<0.05$, log-rank test.

activity in any of the in vitro or in vivo studies described here also argues that the efficacy of TBC3486 in these studies is directly related to its activity toward $\alpha 4 \beta 1$ integrin.

Like many small molecules, TBC3486 is endowed with a rather shorter half-life, requiring more frequent dosing than natalizumab. A shorter half-life should likely be an advantage in leukemia treatment, but challenges at establishing the optimal timing of anti-integrin treatments (how often, how much and so on) relative to the chemotherapy must not be underestimated. Natalizumab led to the complete eradication of pre-B-ALL in our xenotransplant model in two out of three pre-B-ALL cases. By contrast, adjuvant treatment with TBC3486 was associated only with prolonged survival, not with leukemia cell eradication. Although formal sideby-side comparisons were not performed, this may at least in part be due to the differences in treatment schedules (TBC3486: twice daily for 2 weeks, natalizumab: once weekly for 4 weeks), but additional differences of the two compounds may also contribute. As the half-life of natalizumab is much longer, therapeutic drug levels are likely to be continuously maintained for the entire duration of the 4-week VDL cycle.

Taken together, our data demonstrate that small-molecule inhibition of integrin $\alpha 4$ using TBC3486, currently in preclinical evaluation, is a promising approach for targeting chemotherapyresistant leukemia. Further studies are warranted to understand and evaluate preclinically adjuvant small-molecule inhibition of integrins to overcome relapse of ALL.

\section{CONFLICT OF INTEREST}

The authors declare no conflict of interest.

\section{ACKNOWLEDGEMENTS}

The study was supported by the Hyundai Hope on Wheels, V-Foundation, St Baldrick's Scholar Award and NIH R01CA172896. HB acknowledges support from LOEWE OSF TP5a and Deutsche Forschungsgemeinschaft BO3553/1-1.

YT Hsieh ${ }^{1}$, EJ Gang ${ }^{1}$, SN Shishido ${ }^{1}, \mathrm{HN} \mathrm{Kim}^{1}{ }^{1}, \mathrm{~J} \mathrm{Pham}^{1}$, S Khazal ${ }^{1}$, A Osborne ${ }^{1}$, ZA Esguerra $^{1}$, E Kwok $^{1}, \mathrm{~J} \mathrm{Jang}^{1}{ }^{1}, \mathrm{H} \mathrm{Bonig}^{2}{ }^{2}$, RJ Biediger $^{3}$, $\mathrm{P}$ Vanderslice ${ }^{3}$ and YM Kim ${ }^{1}$

${ }^{1}$ Division of Hematology and Oncology, Children's Hospital Los Angeles, University of Southern California Keck School of Medicine, Los Angeles, CA, USA;

${ }^{2}$ Institute for Transfusion Medicine and Immunohematology, JohannWolfgang-Goethe University, Frankfurt, Germany and

${ }^{3}$ Wafic Said Molecular Cardiology Research Laboratory, Texas Heart Institute, Houston, TX, USA E-mail: ymkim@chla.usc.edu

\section{REFERENCES}

1 Konopleva MY, Jordan CT. Leukemia stem cells and microenvironment: biology and therapeutic targeting. J Clin Oncol 2011; 29: 591-599.

2 Hsieh YT, Gang EJ, Geng H, Park E, Huantes S, Chudziak D et al. Integrin alpha4 blockade sensitizes drug resistant pre-B acute lymphoblastic leukemia to chemotherapy. Blood 2013; 121: 1814-1818.

3 Steinman L, Merrill JT, McInnes IB, Peakman M. Optimization of current and future therapy for autoimmune diseases. Nat Med 2012; 18: 59-65.

4 Vanderslice P, Woodside DG, Caivano AR, Decker ER, Munsch CL, Sherwood SJ et al. Potent in vivo suppression of inflammation by selectively targeting the high affinity conformation of integrin alpha4beta1. Biochem Biophys Res Commun 2010; 400: 619-624. 
5 Cannella B, Gaupp S, Tilton RG, Raine CS. Differential efficacy of a synthetic antagonist of VLA-4 during the course of chronic relapsing experimental autoimmune encephalomyelitis. J Neurosci Res 2003; 71: 407-416.

6 Masumoto A, Hemler ME. Multiple activation states of VLA-4. Mechanistic differences between adhesion to CS1/fibronectin and to vascular cell adhesion molecule-1. J Biol Chem 1993; 268: 228-234.

$7 \mathrm{Ni} \mathrm{H}$, Li A, Simonsen N, Wilkins JA. Integrin activation by dithiothreitol or $\mathrm{Mn2}+$ induces a ligand-occupied conformation and exposure of a novel $\mathrm{NH} 2$-terminal regulatory site on the beta1 integrin chain. J Biol Chem 1998; 273: 7981-7987.

8 Engelhardt B. The role of alpha 4-integrin in T lymphocyte migration into the inflamed and noninflamed central nervous system. Curr Top Microbiol Immunol 1998; 231: 51-64.
9 Park E, Gang EJ, Hsieh YT, Schaefer P, Chae S, Klemm L et al. Targeting survivin overcomes drug resistance in acute lymphoblastic leukemia. Blood 2011; 118: 2191-2199.

10 Gang EJ, Hsieh YT, Pham J, Zhao Y, Nguyen C, Huantes S et al. Small-molecule inhibition of $\mathrm{CBP} /$ catenin interactions eliminates drug-resistant clones in acute lymphoblastic leukemia. Oncogene 2013; 33: 2169-2178.

This work is licensed under a Creative Commons AttributionNonCommercial-NoDerivs 3.0 Unported License. The images or other third party material in this article are included in the article's Creative Commons license unless indicated otherwise in the credit line; if the material is not included under the Creative Commons license, users will need to obtain permission from the license holder to reproduce the material. To view a copy of this license, visit http://creativecommons. org/licenses/by-nc-nd/3.0/

\section{MYD88 (L265P) mutation is an independent prognostic factor for outcome in patients with diffuse large B-cell lymphoma}

\section{Leukemia (2014) 28, 2104-2106; doi:10.1038/leu.2014.184}

Diffuse large B-cell lymphoma (DLBCL) encompasses an aggressive and heterogeneous group of malignancies. Gene expression profiling (GEP) unraveled two main subtypes based on their putative cell of origin named germinal center B-cell-like (GCB) and activated $B$-cell-like $(A B C)$, with specific genetic alterations and different clinical outcome. ${ }^{1}$ Recent studies have reported several somatic mutations in the myeloid differentiation primary response gene 88 (MYD88) affecting B-cell lymphomas. Among them, the leucine to proline exchange at position 265 (L265P) is the most recurrent and biologically potent MYD88 variation, being found in about $30 \%$ of ABC-DLBCL but uncommon in GCB-DLBCL. ${ }^{2}$ Here, we have investigated the occurrence of MYD88 L265P mutation in adult patients with DLBCL and its relation to clinical and biological characteristics, including patients' outcome.

A series of 175 retrospective patients diagnosed of DLBCL from July 2000 to July 2013 was selected from our lymphoma registry. To be included in this study, cases were required to have: newly diagnosed DLBCL that had not been previously treated with full clinical data available, treatment with remission intention, enough material for DNA extraction and absence of HIV infection. This study was approved by the local ethical committee and was conducted in accordance with the Declaration of Helsinki. Immunohistochemical staining was performed on formalin-fixed paraffin-embedded sections with the following antibodies: CD20 (clone L26, Dako, Glostrup, Denmark), CD10 (clone 56C6, Novocastra, Newcastle, UK), MUM1 (clone MUM1p, Dako), BCL2 (clone 100, BioGenex, San Ramon, CA, USA) and BCL6 (clone PG-B6p, Dako). MYD88 L265P mutation was assessed by allele-specific oligonucleotide PCR that can detect low levels of mutant DNA as previously described. ${ }^{3}$ Association with clinical characteristics was investigated by Fisher's exact test for categorical data and $t$-test or Mann-Whitney $U$-test for continuous data. Overall survival (OS) and progression-free survival (PFS) were calculated since the time from first treatment and according to standard definitions. Kaplan-Meier survival estimates were obtained and multivariate Cox regression was used to determine whether mutation remained independently predictive of PFS and OS after adjusting for clinical variables. Significance was set at $P \leqslant 0.05$.

The major clinical characteristics of the 175 patients are similar to other series of DLBCL (Supplementary Table 1), with the exception of a slightly higher frequency of primary extranodal cases $\left(43 \%\right.$ in our study vs $20-40 \%$ in literature). ${ }^{4}$ This fact may reflect a particular referral pattern for several lymphoma types in our institution. MYD88 L265P mutation was found in 17 out of 175 cases (10\%), a frequency slightly lower than that initially reported, ${ }^{2}$ although a recent study has shown an even lower incidence $(6.5 \%){ }^{5}$ To the best of our knowledge, there are few studies analyzing the clinical features associated to MYD88 L265P mutation in DLBCL patients. ${ }^{2,5-8}$ Clinical characteristics of our series according to MYD88 mutational status are shown in Supplementary Table 2. MYD88 mutation occurred more frequently in males $(P=0.019)$, cases without B symptoms $(P=0.006)$ and those with primary extranodal disease $(P=0.02)$. In agreement with recent observations, ${ }^{2,5}$ MYD88 mutation was infrequent in DLBCL arising in lymph nodes (3\%), but more frequently found in extranodal sites such as central nervous system (50\%), skin (33\%) and testes (78\%). ${ }^{6,9,10}$ Of note, and in contrast to other studies, we found no mutated cases among gastric, small intestine and large intestine DLBCL. ${ }^{7}$ Therefore, our results confirm the remarkable site-specific occurrence of MYD88 mutations at some immune-privileged locations. As somatic mutations in MYD88 are the most frequent alterations found in $A B C-D L B C L$, we further analyzed $G C B$ or $A B C$ phenotype by immunohistochemistry according to the criteria of Hans. ${ }^{11}$ As previously reported, MYD88 mutations were predominantly observed in the ABC type (14\% vs $6 \%$ in GCB type, $P=0.214$ ). ${ }^{2,7}$

For the whole cohort, the median follow-up time was 41 months (range, 2-171 months), with OS at 4 years of $69 \%$ and PFS at 4 years of $57 \%$. Univariate analysis showed that intermediatehigh or high International Prognostic Index (IPI) $(P=0.001)$ and MYD88 L265P mutation $(P=0.001)$ (Figure 1a) were significantly associated with inferior $O S$, whereas no correlation was found with primary extranodal origin $(P=0.946)$ or $A B C$ phenotype assessed by Hans' algorithm $(P=0.953)$. When adjusted in a multivariate Cox regression analysis, MYD88 L265P mutation remained as a significant risk factor for death (hazard ratio $(\mathrm{HR})=2.4,95 \%$ confidence interval $(\mathrm{Cl}), 1.2-4.7, P=0.013)$ as well as intermediatehigh or high IPI $(\mathrm{HR}=3.0 ; 95 \% \mathrm{Cl}, 1.6-5.7, P=0.001)$. Regarding PFS, MYD88 mutation was a significant factor for inferior PFS (logrank, $P=0.049)$, but not $\mathrm{ABC}$ phenotype $(P=0.956)$. However, our results using the Hans' algorithm for DLBCL subgroup stratification should be taken with caution as algorithms based on immunohistochemistry, even those recently proposed, have variable concordance with GEP.

Most patients were treated according to standard practice but some cases were enrolled into clinical trials or received specific treatments (for instance, primary brain lymphomas were treated 